Cómo citar este trabajo: Clemente Villar, C. (20XX). EL impacto psicológico del estigma prostituta. Revista del Laboratorio Iberoamericano para el Estudio Sociohistórico de las Sexualidades, 04 art. 08, 152-172. https://doi.org/10.46661/ relies.5106

\title{
El impacto psicológico del estigma de prostituta
}

\author{
The psychological consequences of the prostitute stigma
}

\author{
Carolina Clemente Villar \\ Graduada en psicología por la UOC \\ Colectivo de prostitutas de Sevilla \\ carclemvill@gmail.com
}

Resumen

Los diagnósticos que más se repiten en la literatura que analiza la relación entre psicopatología y trabajo sexual son "Depresión", "Trastorno de Estrés Post-Traumático" y "Trastorno relacionado con las sustancias". Acerca de su causalidad, los profesionales de la salud mental suelen extraer conclusiones esencialistas y psicopatologizantes, ya que conciben el trabajo sexual como una actividad inherentemente violenta. Este tipo de lectura, sin embargo, no analiza el papel causal del estigma en el padecimiento de trastornos, mediante diferentes tipos de violencia: la afectación del autoconcepto (baja autoestima y autoeficacia), la afectación de las relaciones interpersonales (aislamiento social y problemas -e incluso maltrato- con el entorno) y violencia institucional (legislaciones punitivistas, atención estigmatizante por parte de los servicios de asistencia y los servicios de salud -incluyendo salud mental- y abandono -pobreza, falta de oportunidades y de alternativas laborales-). El objetivo de este artículo es analizar la causalidad del estigma en la generación, desarrollo y mantenimiento de la Depresión, el TEPT y el consumo de sustancias. La finalidad es que el profesional de la salud mental los tenga en cuenta en el análisis del problema y el diseño del tratamiento, a fin de que éstos sean más acertados y las conclusiones no se basen en estereotipos.

Palabras clave: Prostitución, Estigma, TEPT, Depresion.

Abstract

The most frequently repeated diagnosis in the literature that analyzes the relationship between psychopatology and sex work are Depression, Posttraumatic Stress Disorder and Substance use Disorders. Mental health professionals describe their causation as essentialist and psychopatological conclusions, since they conceive sex work as an inherently violent activity. 
However, this type of analises does not consider the causal role of stigma in suffering from disorders, through different types of violence: affection of self-concept (low self-esteem and decreased selfefficacy), affection of interpersonal relationships (social isolations and problems -even abuse- with the environment) and institutional violence (punitive laws, stigmatizing attitudes by health care services -including mental health-, as well as neglection -poverty, lack of oportunities and job alternatives-). The objective of this article is to analyze the causality of stigma in the generation, development and maintenance of Depression, PTSD and substance use. The aim is mental health professional to consider them in the analysis of the problem and the design of the treatment, so that they are more accurate and the conclusions are not based on stereotypes.

Key words: Prostitution, Stigma, PTSD, Depression. 


\section{Introducción}

El trabajo sexual es una actividad fuertemente discriminada. Conlleva, de manera inherente, un estigma que atraviesa toda su realidad y se manifiesta en tres ámbitos: las instituciones -tanto públicas como privadas-, las relaciones interpersonales y el autoconcepto.

En el ámbito institucional destaca el punitivismo, que comporta clandestinidad, con el consecuente peligro para nuestra integridad física y psíquica. A su vez, el punitivismo incide en el imaginario social, que nos describe como delincuentes o desviadas a las que es lícito castigar. Esto no sólo afecta a nuestro autoconcepto, sino que también legitima la violencia contra nosotras y dificulta que nos empoderemos y nos organicemos políticamente para exigir cambios a nivel institucional. Asimismo, el impacto del estigma en el autoconcepto puede generarnos sentimientos de culpa o de desprecio y, en consecuencia, dar pie a actitudes permisivas con la violencia.

Las consecuencias del estigma de prostituta son, por tanto, fuente de malestar psicológico. Sin embargo, la literatura clínica que trata el tema no suele incluirlas en sus análisis. Más bien, tiende a esencializar la psicopatología como una consecuencia inherente e inevitable del trabajo sexual.

Esta falla en la investigación académica comporta consecuencias cuando acudimos a un profesional de la salud mental: éste puede guiarse por sus prejuicios acerca de que la prostitución es una actividad perjudicial para la salud mental o que se debe a una conducta desviada. En consecuencia, su tratamiento puede no ser ajustado a nuestras necesidades e incrementar el estigma.

Los trastornos que más aparecen en la literatura sobre prostitución y psicopatología -Depresión, TEPT y Trastorno relacionado con sustancias- pueden tener un origen exógeno. Dar por sentado que la prostitución, de manera inherente e inevitable, afecta negativamente a la salud mental puede restar capacidad de agencia a la trabajadora sexual y dónde sitúe su locus de control. Éste es el modo en que un sujeto percibe su influencia en los acontecimientos que le implican. Si cree que éstos dependen de sus actos y sus cualidades, contará con un locus de control interno. De lo contrario, si cree que escapan a su control e influencia, su locus de control será externo. Por ende, las definiciones esencialistas por parte de la clínica, en que el trabajo sexual es descrito como inocivo per se y que incluso psicopatologizan a las trabajadoras sexuales pueden desviar el locus de control de un lugar externo al interno. En consecuencia, disminuirán las acciones, por parte de la trabajadora, dirigidas a cambiar las fuentes de malestar. Con esto, la gravedad del trastorno puede mantenerse o, incluso, incrementarse. Por el contrario, reconocer nuestra capacidad y conciencia de agencia puede resultar un factor clave a la hora de disminuir la gravedad de dichos trastornos.

En este artículo se analiza, a partir de diversos estudios de campo, cuáles son los factores del estigma de prostituta que inciden negativamente en la salud mental y qué estrategias de agencia puede desarrollar una trabajadora sexual frente a ellos. El objetivo es que los profesionales de la salud mental tengan en cuenta esta información durante los tratamientos y que éstos estén dirigidos a cambiar ciertos elementos estresores. En esta línea, se darán algunas claves para la intervención.

La estructura del artículo comprende, en primer lugar, la descripción del estigma de prostituta. En segundo lugar, los datos cuantitativos y cualitativos de los diferentes estudios consultados. Asimismo, se ha analizado, desde una perspectiva de psicología clínica, cómo el estigma contribuye a la generación y mantenimiento de dichos trastornos. Las fuentes consultadas han sido artículos de los siguientes repositorios: Psycharticles, Psychology Database, Psychology \& Behavioral Sciences 
Collection i Psychinfo. También se ha empleado Google Scholar. Se descartaron los artículos cuyas muestras procedieran de contextos de trata. Las palabras clave han sido las siguientes: Prostitution and "Mental Health", Prostitution and "Mental Ilness", Prostitution and Depression, Prostitution and PTSD, Prostitution and Stigma, Prostitution and Mental Health and Meta-Analysis, Prostitution and Empowerment, Prostitution and Harm Reduction. La metodología empleada ha sido el metaanálisis de los estudios mencionados.

\section{Descripción del estigma de prostituta}

Goffman define el estigma como el atributo por el cual "dejamos de verlo [a quien lo posee] como una persona total y corriente para reducirlo a un ser inficionado y menospreciado" (Goffman, 1963). En el caso de las trabajadoras sexuales, esta marca simbólica justifica y normaliza que seamos discriminadas de manera estructural. Lejos de poder acotarse como un mero componente del trabajo sexual, el estigma es el elemento central que vertebra toda nuestra realidad. Más en concreto, opera en tres ámbitos que se interrelacionan entre sí: el autoconcepto, las relaciones interpersonales y las instituciones.

La interiorización del estigma afecta al autoconcepto. Al contrario de lo que sucedería con otros grupos que, siguiendo a Tajfel (Tajfel, 1981, citado en Ibáñez-Gracia et al., 2016), conforman su identidad a través de procesos de categorización social del grupo de pertenencia, las trabajadoras sexuales no solemos tener una relación de afirmación con la misma, sino que la llevamos en secreto

Lo hacemos por las consecuencias que comporta el estigma: la autopercepción negativa (y su consecuente baja autoestima), el sentimiento de inferioridad -que puede conllevar incluso la permisividad de cara al maltrato-, la predisposición al fracaso y el efecto Pigmalión (Ibáñez-Gracia et al., 2016).

Diversos estereotipos contribuyen a esta autopercepción negativa. Uno es la idea de que somos mujeres sucias y deshonradas (Ryan et al., 2019), idea reforzada por el estereotipo de las prostitutas como personas marcadas por las carencias morales (Goffman, 1963, en Trushca, 2016). Para algunas trabajadoras sexuales, su identidad como tal entra en conflicto con su visión de las relaciones íntimas, así como con ciertas características que la sociedad atribuye a un comportamiento femenino aceptable. Por ejemplo, pueden temer la vergüenza que llevarán a sus familias si hacen pública su ocupación (Yuen et al., 2014).

El modo en que nuestra autoestima se ve afectada puede comportarnos sentimientos de inferioridad y repercutir en nuestra autoeficacia (Panduriaru et al., 2011, a Zhang et al. 2017): el estigma de prostituta es deshumanizante $y$, cuando lo interiorizamos, aceptamos esa deshumanización (Gofman, 1963, en Trushca, 2016). Por ello, muchas veces acabamos justificando los prejuicios, la violencia y la discriminación que la sociedad mantiene hacia nosotras, como algo que merecemos: justificamos, entre otras violencias, la falta de respeto y la devaluación (Benoit, 2018). La interiorización del estigma también nos produce sentimientos de desempoderamiento (Benoit, 2018), así como un mayor riesgo de trastorno mental (estrés y ansiedad) y una mayor susceptibilidad de sufrir abusos sexuales (Puri et al., 2017). En consecuencia, la calidad de vida empeora y los trastornos se agravan (Panduriaru et al., 2011, citado en Zhang et al. 2017). El estigma interiorizado también puede comportar sentimientos de culpa, los cuales generan síntomas somáticos (Pandiyan, 2014).

Otro elemento que afecta negativamente al self es el conflicto entre los diferentes roles que desarrollamos en nuestra cotidianeidad. El rol como prostituta se opone a los mandatos sociales que marcan el comportamiento sexual femenino socialmente aceptado. Así, por ejemplo, en China (Zhang et al., 2015), para ser aceptadas, las mujeres tienen que ser amables, pasivas y sexualmente inocentes. En India (Pandiyan, 2014), es incompatible la aceptabilidad social con el sexo 
prematrimonial. El rol como prostituta también puede suponer confrontación con otros roles: uno es el de esposa. Así, en el estudio de Cange (2019) vemos que las mujeres con mayor grado de depresión son aquéllas que están o han estado casadas. Otro es el rol de buena madre (Truschka, 2016; Benoit, 2017). El trabajo sexual también puede generar conflictos y disonancia cognitiva cuando resulta incompatible con la religión (Velez y Audet, 2019).

En ocasiones, puede suceder lo contrario y el buen desempeño de otros roles comporta ventajas, incluso en el entorno del trabajo sexual. Así, en el estudio de Zhang et al. (2015) las mujeres casadas y de mayor edad son las que mantienen una mejor relación con el encargado de la seguridad del prostíbulo. También cuentan con un mayor respaldo emocional: esto les permite reducir el estrés psicológico.

Para solucionar la disonancia cognitiva entre roles, muchas trabajadoras sexuales justifican el ejercicio de su profesión precisamente como una manera inevitable de cumplir con las obligaciones familiares (Yuen et al., 2014; Benoit, 2017). También hacen que la identidad principal -aquélla desde la que se relacionan con su entorno, que lleva el nombre de su documento de identidad- se adapte perfectamente a las demandas sociales relacionadas con la femineidad hegemónica (Benoit, 2017).

En la misma línea, otra de las estrategias es, al construir el autoconcepto, reforzar la identificación con esos roles socialmente aceptados; por ejemplo, el rol de madre o esposa. Con esto, hay un rol que siempre permanece escondido: en terminología de Goffman, cuando se encarna la identidad principal, se esconde el rol como prostituta en el fondo o región invisible para el público y viceversa. No obstante, esta división entre roles no es algo gratuito: comporta la angustia de que, si se descubre la identidad de la región invisible, las circunstancias personales viren hacia la exclusión, la soledad, la marginalidad, etcétera. Asimismo, aunque se lleve en secreto, la identidad como trabajadora sexual es parte nuestro autoconcepto $y$, por ende, influye en nuestra autoestima.

El segundo ámbito que analizo son las relaciones interpersonales. Como se decía, el estigma es deshumanizante (Cange, 2019). En consecuencia, incrementa la victimización, puesto que justifica las agresiones (Comack \& Seshia, 2010, en Štulhofer et al., 2016). Así, las trabajadoras sexuales podemos presentar un mayor índice de violencias padecidas, tanto por parte de nuestra pareja, como de los clientes o la policía (Štulhofer et al., 2016; Zhang et al., 2017).

Se suele justificar la violencia hacia nosotras mediante los estereotipos: por ejemplo, el que nos relaciona con la suciedad, la inmoralidad y la monstruosidad y que provoca sentimientos de autoculpabilización, así como aislamiento social (Zhang et al., 2017). Por ello, la mayoría de trabajadoras sexuales esconde su ocupación a amigos y familiares, por miedo al rechazo (Hong, Li, Barro, \& Zhao, 2007, a Zhang et al., 2015). También suelen experimentar un miedo constante a ser descubiertas, razón por la cual viven una doble vida. Esto es una fuente constante de estrés, depresión y baja autoestima (Yuen et al., 2014). El aislamiento social también afecta a las funciones cognitivas (Cacioppo y Cacioppo, 2012, en Trushka, 2016).

En el caso de desvelar cuál es nuestra actividad, el entorno puede reaccionar con actitudes culpabilitzantes, por no seguir las normas sexuales tradicionales (Randy, 2019). Otras reacciones están relacionadas con el efecto totalizador del estigma: cualquiera de nuestros atributos que coincida con éste hace que se nos atribuya la totalidad del mismo (Goffman, 1963, en Benoit, 2017).

Esto dificulta todo tipo de relaciones, entre ellas, las relaciones de pareja (Pinedo-González y LópezSánchez, 2015), dado que puede generar sentimientos de celos, desconfianza, culpabilidad y recriminaciones relacionadas con las ITS (Bilardi et al., 2010). Por lo tanto, se suele esconder información, con la finalidad de crear la ilusión de fidelidad y evitar conflictos (Trushka, 2016; Benoit, 2017). También pueden darse situaciones de violencia de género (entendida como violencia en el contexto de la pareja): Zhang et al. (2017), afirman que el estigma de prostituta está relacionado 
con mayores niveles de violencia de género y con una mayor prevalencia de trastornos. Una posible reacción ante ésta es el miedo a que el entorno nos prejuzgue y discrimine por haber sido maltratadas (Zhang et al., 2017), cosa que aumenta el aislamiento social. Otra son las ideaciones suicidas (Zhang et al., 2017). A veces, dadas estas dificultades, mantenemos relaciones con los clientes, pero éstas suelen ser muy complicadas, generando estrés (Yuen et al., 2014). .

Otra de las consecuencias del estigma interiorizado es que dificulta la creación de una identidad colectiva, haciendo que el estigma se reproduzca incluso en los burdeles (Ryan et al., 2019). En consecuencia, en nuestro mismo colectivo se da una tendencia a la competencia, en lugar de comportamientos solidarios (Yuen et al., 2014). Como estrategia para mantener la propia legitimidad, se realizan comparativas intergrupales, en que se proyecta el estigma en el otro grupo. Por ejemplo, las que trabajan en interiores con las de calle (Benoit, 2017).

El modo en que el estigma afecta a las relaciones interpersonales acarrea una serie de consecuencias. En primer lugar, el sentimiento de soledad, a causa de los prejuicios y la discriminación (Pinedo-González y López-Sánchez, 2015). A su vez, el aislamiento social da pie a una mayor probabilidad de sufrir violencia por parte tanto de la familia como de la pareja (Zeglin, 2014, citado en Pinedo-González y López-Sánchez, 2015).

Por el contrario, el estrés y el apoyo social correlacionan negativamente (Štulhofer et al., 2016): el estrés se ve reducido por el apoyo social; del mismo modo, una falta de apoyo social aumenta el riesgo de relaciones tóxicas. El apoyo social también disminuye el riesgo de tratamiento psiquiátrico, así como el riesgo de contracción de $\mathrm{VIH}$, puesto que, como se verá más adelante, las conductas sexuales de riesgo pueden estar ligadas a una mala salud mental.

El tercer ámbito que analizo es la violencia institucional. Ésta comporta diferentes aspectos. El primero es legal y se refiere a todas las leyes que criminalizan el trabajo sexual, dificultando su ejercicio. Así, siguiendo a Velez y Audet (2019), la criminalización del trabajo sexual comporta un incremento de la violencia hacia las prostitutas, sobre todo las que captan clientela en la calle: el hecho de tener que ir a zonas más alejadas para evitar a la policía incrementa este riesgo ya que, por ejemplo, reduce el tiempo que tienen para saber si quieren aceptar a un cliente. Perseguir el trabajo sexual implica que aquel sector que está más visible (calle) se verá reducido y, en consecuencia, aumentará la explotación laboral en el sector que trabaja en espacios cerrados. También se limitará la protección por parte de la policía y las posibilidades de recibir apoyo por parte de entidades de asistencia. La penalización también aumenta las estancias en la institución penitenciaria (Štulhofer et al., 2016) y el acoso policial (Puri et al., 2017).

Las repercusiones del punitivismo son mayores en el trabajo sexual de calle. El espacio de calle también está relacionado con factores de clase social y de etnia. Así, existen notables diferencias entre los colectivos que trabajan en interior (etnia blanca, mujeres cis y clase media) y los que trabajan en el exterior (minorías étnicas y mujeres transexuales). Estas segundas son las que sufren más cantidad de violencia (Vélez 2019).

Así pues, hay diversos factores estructurales que afectan todavía más a las trabajadoras que pertenecen a colectivos minoritarios. En primer lugar, ser una persona migrante. Esto comporta un mayor nivel de indefensión, por varios motivos: la soledad (Pinedo-González y López-Sánchez, 2015), no tener suficientes herramientas lingüísticas para lidiar con los clientes (Rössler, 2010), no tener suficientes recursos lingüísticos ni conocimientos como para recibir apoyo de los servicios sociales (Rössler, 2010) y la obligación de pagar deuda migratoria, cosa que les impide ser más selectivas con los clientes (Rössler, 2010). En segundo lugar, pertenecer al colectivo LGTBI. Las personas transexuales, por ejemplo, se ven especialmente afectadas por desigualdades sociales y sanitarias. También sufren violencia traumática ligada a la transfobia y la criminalización. Suelen haber disfrutado de pocas oportunidades y, en consecuencia, tienen bajos niveles educativos: esto 
da pie a tasas altas de depresión (Puri et al., 2017). El estigma de la prostitución acompañado del estigma de la LGTBI-fobia también se da en los hombres trabajadores sexuales quienes, además de afrontar el estigma de la prostitución, también afrontan el de la homosexualidad (Benoit, 2017). En tercer lugar, pertenecer a una minoría étnica: por ejemplo, algunos sectores de la industria del sexo en Canadá son altamente racializados, con personas de ascendencia indígena. A causa del racismo, han sufrido más abusos físicos, emocionales y sexuales que sus compañeras blancas.

Otra consecuencia del estigma, a nivel legal, es que nuestra actividad no se reconozca como trabajo $y$, en consecuencia, que no contemos con derechos laborales. Esto se traduce en largas jornadas de hasta 24 horas, que no sólo suponen agotamiento y afectan a la salud mental (Vidal, 2014), sino que también dificultan que mantengamos relaciones sociales (Pinedo-González y López-Sánchez, 2015). Además, algunas modalidades de trabajo sexual suponen un cambio constante de ciudad, cosa que impide establecer vínculos (Pinedo-González y López-Sánchez, 2015).

La violencia institucional también se traduce en el abandono: la falta de oportunidades y, en consecuencia, la pobreza. La mayoría no tenemos otra fuente de ingresos y los salarios que podríamos encontrar en otra actividad económica son bajos (Goretti-laisuklang i Ali, 2017). Esto se debe también al nivel de estudios, el cual corresponde a la falta de oportunidades (Vidal, 2014). Asimismo, la criminalización del trabajo sexual en ciertos países supone un expediente policial, que dificulta encontrar un alojamiento o un trabajo.

Otro ámbito de violencia institucional son las entidades que nos dan asistencia. Las ONGs pueden dar un trato discriminatorio, incluyendo el lenguaje que, en ocasiones, emplean cuando se refieren a nosotras -términos como "puta" o "mujer sucia" (Randy, 2019)-. También dan por sentada y nos devuelven una mirada victimizante, corroborando el prejuicio social de que somos objetos pasivos. Esto contribuye a reducir nuestra capacidad de agencia (Dodsworth 2014, citado en Truschka, 2016).

La violencia institucional también se refleja en la violencia policial, la cual representa muchas veces una clara violación de nuestros derechos: acoso verbal, humillación pública, exceso de fuerza, conductas invasivas, detenciones injustificadas, imposición de pagos, violaciones (Benoit, 2017). Asimismo, cuando la policía identifica a una mujer como prostituta, esto acarrea consecuencias a largo término. Por ejemplo, intervenciones policiales, acoso y humillación, incluso en los momentos en que no esté trabajando (Benoit, 2017). La violencia policial también aumenta nuestra indefensión: para evitar que la policía tenga constancia de nuestro trabajo, muchas veces dejamos de denunciar las agresiones que padecemos.

La discriminación también tiene lugar en los servicios de salud, donde se nos ve como portadoras de VIH. Por ello, en ocasiones, los sanitarios toman con nosotras más medidas o distancia que con otros pacientes e, incluso, dudan si tocarnos. También nos acusan de ser codiciosas y que eso incremente la probabilidad de que nos infectemos de alguna ITS (Ryan et al., 2019). Muchas veces presentan una actitud ambivalente, con una cierta reticencia a tratarnos, que sólo superan por el sentido del deber profesional (Benoit, 2017). Hay incluso quienes, en algunos casos, se han negado a proporcionarnos atención. La consecuencia de este trato estigmatizante y del estigma social es que muchas veces las trabajadoras sexuales no utilizamos los servicios de salud (Puri et al., 2017; Benoit, 2017): los sentimientos de culpa y vergüenza actúan como barreras a la hora de acudir a los mismos (Velez y Audet, 2019). Esto aumenta el riesgo de sufrir VIH y otras ITS. Resulta indicativo que, en los países donde el trabajo sexual se ha despenalizado completamente, los niveles de contagio de ITS son menores (Štulhofer et al., 2016).

En cuanto al sistema de salud mental, en él no es infrecuente el trato discriminatorio: el profesional puede mostrar hacia nuestro trabajo una actitud desdeñosa y carente de apoyo. También directiva y poco comprensiva hacia nuestras circunstancias materiales (Vélez, 2019). Las actitudes discriminatorias que las trabajadoras sexuales podemos encontrar en este ámbito son: lenguaje 
abusivo, trato irrespetuoso, humillación, negación de atención y falta de confidencialidad (Benoit, 2017).

La consecuencia es que muchas trabajadoras sexuales nos negamos a pedir atención psicológica, incluso si la necesitamos (Velez y Audet, 2019). Asimismo, los profesionales de salud mental suelen demostrar un vacío de conocimiento acerca de nuestro trabajo: nos encontramos con que tenemos que invertir energía en informarles (Velez y Audet, 2019). Además, muchas veces la terapia no resulta exitosa, precisamente por la información que no damos por miedo a ser estigmatizadas (Benoit, 2017). Por tanto, para que un profesional de la salud mental proporcionase un tratamiento adecuado, tendría que tener un conocimiento satisfactorio sobre trabajo sexual y sobre la heterogeneidad de realidades que tienen lugar en él. También debería dejar de lado sus prejuicios (Velez y Audet, 2019).

Por el contrario, una atención psicológica no estigmatizante reduce el estigma interiorizado y el sentimiento de vergüenza (Zendher et al., 2019). En aquellos casos en que sí ha habido respaldo por parte del terapeuta, esto ha ayudado en los siguientes aspectos: sentimiento de liberación de la carga, mitigación de conflictos internos relacionados con el trabajo sexual -cosa que facilita el avance de la terapia- y un mayor sentimiento de confianza hacia el terapeuta (Velez y Audet, 2019).

\section{El impacto del estigma de prostituta en la salud mental}

Las diversas violencias que se han descrito conllevan un impacto negativo en la salud mental. En este artículo, pretendo analizar sus repercusiones en la generación o cronificación de la Depresión, el TEPT o el Trastorno relacionado con las sustancias, dado que son los diagnósticos que más se repiten en la literatura sobre prostitución y psicopatología (Cange, 2019; Carlson et al., 2017; Coetzee et al., 2018; Goretti-laisuklang y Ali, 2017; Krumrei-Mancuso, 2017; Pandiyan et al., 2014; Pinedo-González y López-Sánchez, 2015; Rogers et al., 2015; Teixeira y Oliveira, 2016; Vidal, 2014; Zhang et al., 2017; Ziao y Bungay, 2018).

Las violencias derivadas del estigma no siempre se tienen en cuenta desde la clínica: la falta de conocimiento real acerca del trabajo sexual puede comportar que el psicólogo clínico dé por hecho que el malestar proviene del intercambio de sexo por dinero. Estas presuposiciones -inherentes a los prejuicios sobre prostitución- suponen el riesgo de que, al dictaminar las conductas problema y diseñar el tratamiento, se dejen de lado factores importantes. Lejos de querer descartar que el sexo por dinero sea, en sí mismo, una causa de malestar en ciertos sujetos, lo que propongo con este artículo es que se puedan encontrar otros factores adicionales, para que también sean tenidos en cuenta. Por ello, para indagar en la causalidad de los tres trastornos mencionados, he analizado el papel del estigma.

\subsection{El estigma de prostituta y la depresión}

En este apartado analizaré cómo las violencias derivadas del estigma pueden generar o contribuir a una depresión, valiéndome de las siguientes formulaciones teóricas: el enfoque comportamental de Lewinsohn (Lewinsohn et cols., 1979, citado en Vallejo-Pareja, 2015), el enfoque del autocontrol de Rehm (Rehm, 1977, 1981 i 1988, citado en Vallejo-Pareja, 2015), la teoría de la indefensión aprendida de Seligman (Selgiman, 1975, citado en Vallejo-Pareja, 2015) y el enfoque cognitivo de Beck (Beck, 1967, citado en Vallejo-Pareja, 2015).

Una de las hipótesis que sostengo es que las legislaciones punitivistas favorecen la depresión. Así, la criminalización, al provocar clandestinidad, incrementa las agresiones hacia nuestro colectivo, a la vez que dificulta o imposibilita que las denunciemos. Pese a que relaciono este nivel de violencia con el origen de una depresión, los datos cuantitativos de los estudios que he consultado no permiten establecer una relación directa entre ambos factores. 
Vemos cómo, en algunos casos, la criminalización total del trabajo sexual coincide con los altos niveles de depresión: 41,8\% en el estudio de Cange en Burkina Fasso (2019); 60,4\% en el estudio de Carlson et al. (2017) en Mongolia (60,4\%) y 68,7\% el estudio de Coetzee (2018) en Sudáfrica. En otros, en cambio, no: el estudio de Zhang et al. (2017) se desarrolla en China, que es un país prohibicionista y sólo registra un $17,33 \%$ de casos.

La criminalización de la compra de servicios sexuales presenta resultados ambivalentes: en algunos casos, coincide también con altos niveles de depresión, como el estudio de Rogers, Lemstra y Moraros (2015) en Canadá (65,9\%); en otros estudios realizados también en Canadá, la prevalencia de depresión es menor: Puri et al., (2017) presentan unos resultados del 35,1\%.

Los resultados suelen presentar una prevalencia menor en algunos países donde el trabajo sexual en ámbito de interior no está penalizado: un 9\% en el estudio de Goretti-laisuklang i Ali, 2017, en la India o un 33\% con un 10\% de alto riesgo de suicidio en el de Pandiyan, también en la India (2014). No obstante, el estudio de Teixeira (2017) en Portugal -donde la legislación no es penalizadora-, muestra una elevada prevalencia de sintomatología depresiva $(46,15 \%)$.

Dado que el dato no muestra correlación entre los niveles de depresión y el tipo de legislación de cada país, he analizado los datos cualitativos. Éstos presentan las siguientes causas de depresión: violencia física, discriminación por ser prostitutas y bajos niveles de autoestima (Coetzee, 2018), así como las agresiones sexuales (Vidal, 2014). También figuran, entre dichas causas, los embarazos no deseados como consecuencia de una violación (Zhang et al., 2017). Otras causas de estrés psicológico ligadas al trabajo sexual son: miedo a la infección, llevar una doble vida, problemas con la pareja, ciertas demandas de los clientes, problemas sexuales, el trabajo en sí, sentimientos de vergüenza y de culpa y malas condiciones de trabajo (Rössler, 2010). También se mencionan: imposibilidad de controlar la situación, inestabilidad económica, riesgo de contagiarse de una ITS y demandas poco razonables por parte de los clientes (como no utilizar preservativo) (Yuen et al., 2014). En el estudio de Carlson et al. (2017) vemos las siguientes: número de clientes, violencia por parte de éstos, uso del preservativo, preocupaciones sobre el dinero, uso de alcohol, riesgo de violencia sexual, percepción del estigma, acceso reducido a los servicios de salud y carencia de apoyo social. La pertenencia a un colectivo minoritario también incrementa la posibilidad de sufrir un trastorno, debido a la mayor violencia que se vivencia (Puri et al., 2017): tal y como se ha explicado en el apartado "Descripción del estigma de la prostitución", una condición marginal conlleva desde violencia institucional, hasta violencia por parte del otro y repercusiones negativas en el autoconcepto.

Podemos observar que, en muchos de estos factores -extremadamente aversivos-, el locus de control es externo: la violencia física, la discriminación, las violaciones y los consiguientes embarazos no deseados, las infecciones o la inestabilidad económica son ejemplos de ello. Siguiendo la teoría de la indefensión aprendida de Seligman, este locus de control externo sería una importante causa de depresión. Las legislaciones punitivistas incrementan la desprotección de cara a estas violencias y, por tanto, la indefensión.

La concepción criminalizadora del trabajo sexual, propia del estigma, es el pilar de las legislaciones punitivistas. El estigma también justifica las agresiones hacia mi colectivo, puesto que nos deshumaniza. Por tanto, el estigma estaría en el origen del estilo atributivo negativo, propio de la indefensión aprendida y de la desesperanza que, a raíz de padecer las violencias mencionadas, pueden desarrollar algunas trabajadoras sexuales.

El estigma hace que nos autopercibamos como culpables y meritorias del castigo social. En consecuencia, tanto la auto-observación como la auto-evaluación serán muy peyorativas y comportarán tasas bajas de auto-refuerzo (Rehm, 1977, 1981 i 1988, citado en Vallejo-Pareja, 2015) 
Por tanto, la prevalencia de la depresión entre las trabajadoras sexuales puede ser superior a la media de la población femenina (Carlson et al., 2017).

La situación de indefensión ante estas violencias puede derivar en ideaciones suicidas. Éstas son inversamente proporcionales al respaldo social (Teixeira, 2017): los problemas con la familia y la pareja son uno de los factores que comportan un mayor riesgo de ideación suicida. Otra de sus causas son las agresiones sexuales (Cange, 2019), ya sean en el contexto de trabajo o por parte de la pareja (Pandiyan, 2014). Carlson et al. (2017) afirman que las agresiones sexuales son una causa de depresión más significativa que las agresiones físicas. Habría que preguntarse por qué estos dos estímulos aversivos no tienen las mismas repercusiones en la salud mental. Se tendría que estudiar si es porque la violación conlleva un estigma debido a la autoinculpación, puesto que el contexto la atribuye a la conducta de la mujer. También habría que analizar si está relacionada con el estigma de prostituta; es decir, que piensen que la merecen y la han provocado por su comportamiento sexual.

Las ideaciones suicidas también varían en función de quién es el autor de la agresión: en el estudio de Zhang et al. (2017), cuando el agresor es un cliente, las trabajadoras han desarrollado más estrategias para no interiorizar el estigma y para evitar futuras violencias de carácter similar: por ejemplo, no volver a aceptar a dicho cliente. En cambio, cuando la violencia es perpetrada por una pareja estable, aparecen las ideaciones suicidas. Habría que preguntarse si esta mayor afectación se debe a que la relación con la pareja se establece desde su identidad principal y esto tiene un mayor peso para la autoestima. También habría que estudiar otro factor: si la relación con la pareja constituye un factor de peso porque confirma su buen desempeño de los roles socialmente aceptados. Es decir que, en tanto que esa relación permitiría construir la identidad desde un lugar desestigmatizado, se establece una dependencia elevada y, por tanto, una mayor vulnerabilidad.

Esta vulnerabilidad hacia las relaciones sociales puede explicarse desde la teoría de la tríada cognitiva de Beck (Beck, 1967, citado en Vallejo-Pareja, 2015). Si tenemos en cuenta que el estigma incide negativamente en dos de los factores de la triada -la autoimagen y la visión del futuro, mediante la anomia-, esto puede aumentar la dependencia hacia el tercer factor -las relaciones interpersonales-, como protector de cara a la depresión. Esta dependencia puede incrementar una posición de vulnerabilidad y, en consecuencia, el riesgo de sufrir maltrato.

Además de las ideaciones suicidas, existen otros factores ligados al estigma, que pueden dar pie a conductas autodestructivas. En primer lugar, las conductas sexuales de riesgo: las trabajadoras sexuales que han sufrido un mayor número de agresiones sexuales y que más se sienten rechazadas socialmente son las que tienen una mayor tasa de conductas sexuales de riesgo (Pando et al., 2013, citado en Štulhofer et al., 2016; Carlson et al., 2017). Otros factores que inciden en estas conductas son: vergüenza, falta de confianza, indefensión aprendida, infravaloración de los riesgos y desprecio hacia el propio futuro (Štulhofer et al., 2016). En segundo lugar, el estado de ánimo depresivo también está relacionado con el consumo de sustancias. En tercer lugar, la depresión también reduce la efectividad de los programas de salud, como el tratamiento de antirretrovirales (Carlson et al., 2017); seguramente, por falta de adherencia. En cuarto lugar, se ven afectados la autoestima, el sentido de la autoeficacia y del control sobre la propia vida, la percepción del mundo y la confianza en los otros (Macmillan, 2001, en Štulhofer et al., 2016).

Son necesarios otros estudios que analicen en profundidad las conductas autodestructivas que se acaban de nombrar. Más concretamente, la relación que se establece entre la autoimagen deteriorada -consecuencia del estigma de prostituta- y la baja tasa de refuerzo que esto comporta (Lewinsohn et cols., 1979, citado en Vallejo-Pareja, 2015). En el mismo sentido, también habría que estudiar cómo la falta de confianza hacia el futuro y una visión negativa del mundo (que forman parte de la tríada cognitiva) repercuten en este tipo de actitudes. Otro factor que es necesario 
analizar con respecto a las conductas autodestructivas es la indefensión aprendida. Es decir, cómo la privación de herramientas -por ejemplo, legales o simbólicas- de cara a las circunstancias adversas, estaría en el origen de esquemas cognitivos que perpetuarían la pasividad ante estos $u$ otros estímulos adversos.

\subsection{El estigma de prostituta y el Trastorno de Estrés Postraumático}

En este apartado analizaré cómo las violencias debidas al estigma pueden dar lugar a un TEPT, aumentar su gravedad o cronificarlo.

Para establecer un diagnóstico de TEPT, la persona tiene que haber experimentado una situación traumática que implique riesgo de muerte o amenaza a su propia integridad física. También puede haber sido testigo de cómo esto le sucedía a otra persona. Otra causa de TEPT es la violencia sexual (American Psychiatric Association, 2014).

En cuanto a las relaciones sexuales en un contexto de prostitución, para que fueran traumáticas a un nivel de TEPT, tendrían que comportar claramente una amenaza para la propia vida o una situación de violencia sexual. Esto puede darse en un contexto de trata de personas con finalidad de prostitución forzada y en otros contextos de coacción, pero no se puede generalizar como algo propio de todo el sexo comercial. La mera ausencia de deseo en las relaciones sexuales puede tener otras repercusiones, pero no constituye un trauma con la intensidad propia de un TEPT.

Los datos cuantitativos encontrados en los diferentes estudios consultados en relación al Trastorno de Estrés Postraumático son los siguientes: en el estudio de Coetzee -realizado en Sudáfrica-, el $39,6 \%$ presenta sintomatología de TEPT y el 32,7\% la presentaba de manera comórbida con la depresión; en el estudio de Goretti-laisukl i Ali, (2017) -realizado en la India-, el 21\% presenta sintomatología de TEPT. Vemos que la diferencia de legislaciones de los países de ambas muestras -Sudáfrica, donde la venta de servicios sexuales está penalizada y la India, donde es legal en espacios interiores- repercute en la prevalencia de TEPT: bajo la legislación punitivista, es de casi el doble y, por tanto, significativa.

Podemos plantearnos, por tanto, que la legislación sobre prostitución tiene repercusiones relacionadas con el TEPT. Las leyes que penalizan cualquier componente del trabajo sexual -ya sea su ofrecimiento, contratación o el espacio en el que esto se lleva a cabo- llevan nuestro trabajo a la clandestinidad. Esto dificulta o, directamente, imposibilita que denunciemos las agresiones que padecemos, ya que no queremos constar como prostitutas en la denuncia. En consecuencia, las agresiones permanecen impunes, dando más poder a los perpetradores. Por tanto, cuando ejercemos bajo legislaciones punitivistas, las trabajadoras sexuales tenemos más posibilidades de padecer las violencias que desencadenarán un TEPT, es decir, violencias que pongan en riesgo nuestra integridad física o supongan riesgo de muerte.

No obstante, experimentar este tipo de experiencias traumáticas no siempre da pie a un TEPT, puesto que pueden intervenir los siguientes factores protectores: la resiliencia, el temperamento y el apoyo social. Un ambiente libre de estímulos aversivos también previene contra la instalación del trastorno (Vallejo-Pareja, 2015). Las violencias debidas al estigma, por tanto, entorpecen esta recuperación: en primer lugar, como ya se ha dicho, la clandestinidad fomenta la violencia. Asimismo, la violencia simbólica alienta y justifica que se nos agreda.

El impacto negativo del estigma en las relaciones interpersonales también contribuye a que se desarrolle este trastorno. La razón es que, ante un trauma propio del TEPT, el apoyo social puede actuar como un protector para superar dicho trauma y que éste no se desarrolle.

El estigma también afecta a otro factor protector: la resiliencia. Ésta también es necesaria para que no se instale el trastorno a raíz de los traumas propios del TEPT. Sin embargo, el estigma de prostituta hace que la sociedad vea en nosotras personas destruidas y destrozadas y que nosotras, 
a su vez, interioricemos esta imagen. La autoimagen de persona destruida es, asimismo, una de las características diagnósticas del TEPT (American Psychiatric Association, 2014). En consecuencia, a través de la mirada estigmatizante que nos devuelve el otro, no sólo aumenta nuestra autoimagen de persona autodestruida: esta autoimagen también nos resta capacidad de resiliencia y, por tanto, aumenta la posibilidad de que desarrollemos un TEPT.

También inciden en el TEPT las creencias negativas; más en concreto, la percepción del mundo como un lugar peligroso para vivir (American Psychiatric Association, 2014), percepción que resulta comprensible por la violencia que comporta el punitivismo.

En cuanto a la conducta temeraria o autodestructiva que puede aparecer en el TEPT (American Psychiatric Association, 2014), podría ser fruto de la autoimagen estigmatizada. Es decir, en caso de que una trabajadora sexual se viera a sí misma como dictamina el estereotipo: indigna y meritoria de castigo y malestar. Esto, a su vez, podría incrementar la autoimagen de persona destruida. Asimismo, la anomia a la que puede abocar un estigma de prostituta coincide con el sentimiento de ausencia de futuro, característico del TEPT.

Krumrei-Mancuso (2017) encontró una serie de factores que actúan como protectores del TEPT y otros que motivan la sintomatología del mismo. Así, el desarrollo del TEPT correlaciona negativamente con los siguientes factores: tiempo en la actividad, adaptación al trabajo sexual, no haber entrado en éste por necesidad $y$, por último, calidad de vida (auto-realización, autoaceptación, relaciones íntimas, ser aceptada en las relaciones interpersonales, sentimiento de ser tratada de una manera justa por la sociedad, sentimiento de contribución a la misma y religión). Estos factores señalan que la persona tiene un locus de control interno, tanto en su trabajo como en su vida.

En cuanto a los factores que motivan la sintomatología de TEPT, Kumrei-Mancuso (2016) encontró los siguientes: los sujetos que habían empezado en el trabajo sexual por diversión y excitación presentaban menos sintomatología depresiva y de TEPT que los que habían entrado por necesidad económica. Otros factores eran el deseo de abandonar esta actividad y la falta de autoaceptación. También querer dejar este trabajo, pero no tener otras posibilidades o tener poca confianza en encontrarlas. Asimismo, no tener un sentimiento de auto-trascendencia (entendida como la capacidad de contribuir a hacer del mundo un lugar mejor).

Es decir, que el riesgo de TEPT se incrementa cuando el trabajo sexual es una realidad inevitable en la que no se desea estar. $O$, en otras palabras, una realidad que corresponde a los esquemas cognitivos de la indefensión aprendida, donde el locus de control es externo. No descarto que uno de los motivos por los que una trabajadora sexual rechace su trabajo sea el sexo sin deseo a cambio de dinero. No obstante, en tanto que los estudios consultados no aportan información acerca de esto, analizaré los datos de dichos estudios y su relación con el estigma.

Como acabamos de ver, la visión del trabajo sexual como lugar aversivo se debe, entre otros factores, a la falta de oportunidades. Esto corresponde a una de las violencias señaladas en el apartado "Descripción del estigma de prostituta": el abandono institucional, el cual se traduce en falta de oportunidades y de alternativas laborales económicamente dignas. El otro factor nombrado es la falta de autoaceptación, la cual puede deberse a la falta de apoyo social, así como a una autoimagen de persona destruida. Ésta puede ir acompañada de sentimientos de culpa y de vergüenza, es decir, la idea de que merecemos las violencias padecidas por nuestra condición de trabajadoras sexuales. Esto incrementaría las actitudes de indefensión de cara a las distintas circunstancias adversas que se presenten.

Otra causa de TEPT que mencionan los estudios consultados son los abusos sexuales y la violencia en la infancia. Según Rogers et al. (2015), haber sufrido abusos sexuales en la infancia correlaciona 
positivamente con intercambiar sexo por dinero o por drogas en la edad adulta. Es decir, que habría una relación directa entre la violencia sexual y la entrada en el trabajo sexual. No obstante, para Truschka (2016), el destino de la mayoría de personas que han sufrido abusos sexuales en la infancia no ha sido el trabajo sexual. Por tanto, en los casos a los que se refieren los estudios como el de Rogers et al. (2015), la prostitución habría sido una estrategia de supervivencia, en su huida de un medio familiar hostil. En consecuencia, más que el abuso sexual en sí mismo, lo que propiciaría la entrada al trabajo sexual habría sido la falta de vivienda y de medios materiales. En estos casos encontramos también que la falta de habilidades psicológicas para afrontar los abusos en la infancia comporta que la persona busque aprobación y afecto. Por ello, la versión que mantendrá -para ser socialmente aceptada- es que la forzaron a entrar al trabajo sexual (Dodsworth, 2014, en Truschka, 2016).

\subsection{El estigma de prostituta y el Trastorno relacionado con las sustancias}

Los datos sobre consumo de sustancias de los artículos consultados son los siguientes: Gorettilaisuklang i Ali (2017) exponen que, en la muestra de trabajadoras sexuales indias que consultó, el $8 \%$ presentaba dependencia de alcohol, en tanto que el 3\% presentaba dependencia de sustancias psicoactivas; en el estudio de Vidal (2014), realizado con trabajadoras sexuales de Portugal, más de la mitad de la muestra bebe alcohol una vez por semana -en comparación con el 39\% de la población femenina- y un 18\% consume drogas; en el estudio de Yuen et al. (2014), llevado a cabo con trabajadoras sexuales chinas, el consumo es minoritario.

Las diferencias en el consumo de sustancias entre países pueden deberse a los roles de género y su incompatibilidad, a nivel cultural, con la ingesta de alcohol o el consumo de drogas: vemos que las mujeres que más sustancias consumen pertenecen a países europeos, donde este consumo, por parte del género femenino, está normalizado.

Las violencias que padece nuestro colectivo constituyen un factor de riesgo para el Trastorno relacionado con sustancias: la victimización aumenta el riesgo de abuso de drogas y alcohol (Štulhofer et al., 2016). Asimismo, el consumo de sustancias correlaciona positivamente con los síntomas depresivos (Carlson et al., 2017). Para los sujetos con sintomatología depresiva, el consumo de drogas inyectables puede comportar conductas de riesgo, como el intercambio de jeringuillas (Rogers, 2015). En algunos casos, el alcohol se utiliza como ayuda para ejercer la prostitución (Carlson et al., 2017). El consumo de sustancias también puede servir, en otros casos, para enmascarar los efectos negativos de la venta de sexo: estrés y ansiedad (Goretti-laisuklang i Ali, 2017). Puri et al., (2017) establecen una clara relación entre el trauma y el consumo de sustancias: dado que, como se ha dicho antes, la falta de apoyo social y la atención inadecuada por parte de los servicios de salud mental pueden cronificar los efectos de un trauma, el alcohol y las drogas se utilizarán como atenuantes.

Otros factores propios del trabajo sexual que pueden dar lugar a un Trastorno relacionado con sustancias son los siguientes: el elevado número de horas que la trabajadora pasa esperando, la inestabilidad económica, la competencia, la exposición a la violencia física y verbal, el riesgo de robo y la falta de control (Yuen et al., 2014). Algunas de estos factores causales -exposición a la violencia, riesgo de robo y falta de control- son consecuencia del punitivismo, el cual deja a nuestro colectivo en una situación de desprotección. Otros, como los horarios prolongados, son consecuencia de la falta de derechos laborales.

El hecho de captar clientela en el espacio público puede comportar un factor de riesgo para el Trastorno relacionado con sustancias (Rössler, 2010). Esto se debe a que la criminalización sobre el sector de la calle siempre es más fuerte $y$, en consecuencia, las mujeres experimentan más dificultades para denunciar las violencias que padecen. Es decir: sufren una mayor indefensión, 
quedando más expuestas. Así, para Krumrei-Mancuso (2017), en los contextos de prostitución, existe una correlación entre la psicopatología y la violencia recibida.

\section{Estrategias de empoderamiento}

Las estrategias de empoderamiento son todas aquellas habilidades y capacidades psicológicas gracias a las cuales un sujeto cambia, dentro de sus posibilidades, aquellos factores de la realidad que le resultan adversos. Para analizar cómo se produce el empoderamiento, se han consultado estudios con muestras de contextos muy diferentes, con distintos grados de violencia.

La primera es una muestra de prostitutas chinas (Yuen et al., 2014). Una estrategia que emplean es la construcción de una autoimagen positiva. Este nivel de autoestima elevado comporta, a su vez, una buena autoeficacia, -así como extraversión y optimismo-. La autoeficacia -o capacidad de controlar el propio comportamiento- correlaciona negativamente con las secuelas por sucesos traumáticos (Sossou et al, 2008, en Yuen et al., 2014), ya que conlleva un mayor control frente a los estímulos estresores y aversivos. La autoeficacia elevada está relacionada con el tiempo que una persona lleve ejerciendo el trabajo sexual, ya que éste le proporciona un mayor conocimiento y control de las situaciones que pueden darse. Del mismo modo, las mujeres de la muestra que utilizan estrategias de afrontamiento adaptativas también sufren de un menor estrés.

Otro recurso que emplean es la desestigmatización de su actividad: la describen como cualquier otra ocupación, donde no hacen daño a nadie. Así es cómo pueden superar el sentimiento de culpa. La visión de sí mismas y de su ocupación como algo positivo les ayuda a tener más control sobre sí mismas y su entorno, empoderándolas también de cara al uso del preservativo.

Otra estrategia es la búsqueda de motivaciones y de planes de futuro. Para ello, conciben el trabajo sexual como algo temporal. Así consiguen incrementar su esperanza y actitud optimista y, en consecuencia, disminuir los niveles de estrés.

La segunda muestra está conformada por un grupo de hombres trabajadores sexuales de Canadá (Ziao y Bungay, 2018). La primera de las estrategias que emplean está relacionada con el autoconcepto y es la reconceptualitzación del trabajo sexual como algo positivo, dándole un valor pro-social. Otra de las estrategias es establecer unos límites claros con la clientela, para preservar su vida privada. También son selectivos con los clientes y exigen el uso del preservativo. En cuanto al rechazo social, algunos optan por la doble vida. En cambio, otros dan un significado positivo a este rechazo, como un filtro de personas con quien no merece la pena relacionarse. Algunos también solucionan el aislamiento estableciendo contactos positivos con otros hombres trabajadores del sexo.

La tercera muestra está conformada por un conjunto de mujeres que ejercen la prostitución en Australia (Smith, 2016). Sus estrategias son diversas: personalizar sus servicios (por ejemplo, adaptándolos a filosofías orientales) o tomar un papel activo, para que la prostitución no tenga repercusiones negativas en su salud mental.

Sardana et al. (2016) recogen las narraciones de varias prostitutas de la India, de contextos rurales, que han sufrido situaciones muy aversivas: matrimonios forzosos, maltrato por parte de parejas, pobreza, acoso policial o alcoholismo en algún familiar. Los recursos que utilizan son, en primer lugar, la religión, que puede funcionar como una catarsis. Otro recurso -aunque algunas lo rechazan porque les produce desconfianza- es encontrar apoyo dentro del mismo colectivo de prostitutas, compartiendo sus experiencias. En este sentido es importante la figura del educador de pares, una figura de apoyo ante el estrés. El tercer recurso es la formación: educación en salud reproductiva y sexual y campañas de uso del preservativo. También apoyo para establecerse como prostitutas autónomas. 
Weitzer (2017), partiendo de la conceptualización del estigma que realizan Link y Phelan (Link y Phelan, 2001, en Weitzer, 2017), enumera las siguientes estrategias: "recalcar que tuvo plena agencia cuando entró en la prostitución y que tiene un control total en sus interacciones con los clientes, definir la prostitución como una profesión como cualquier otra, negar el daño, condenar a los estigmatizadores o distinguirse de otras personas que ejercen la prostitución en una posición más baja de la jerarquía".

Lim et al., (2015) hicieron un estudio con prostitutas de Camerún, las cuales habían desarrollado varias estrategias para evitar situaciones de violencia: examinar a los clientes antes de ofrecer el servicio -para deducir si serían sujetos peligrosos-, así como el espacio donde lo ofrecerán, para prevenir el peligro. También cobrar los servicios antes de empezar. Cuando se presentan situaciones conflictivas y la negociación no es posible, priorizan su integridad antes de que el dinero. También establecen estrategias colectivas de seguridad con otras prostitutas, procurando atender a los clientes en espacios que no estén alejados de otras compañeras. Asimismo, contratan a hombres que les proporcionen seguridad (a los que denominan "big brother"). Otra estrategia que emplean es mantener una relación con un cliente (al que llaman "le boy"), con quien tienen relaciones sexuales sin profilaxis, a cambio de protección. Esta medida, sin embargo, puede comportar infección de ITS.

Ayuste et al. (2015) describen sus funciones como trabajadoras sociales que atienden a trabajadoras sexuales. Este equipo busca tres tipos de metas. La primera es que la trabajadora desarrolle un sentido de la autonomía, mediante un programa de solución de problemas. La segunda es la toma de responsabilidad: la intención de involucrarse personalmente en su propio avance. La tercera es que la trabajadora tome conciencia de sus capacidades y habilidades, aumentando su autoconfianza.

Benoit, et al. (2016), a raíz del análisis de diferentes artículos sobre prostitución, definen los tres pilares de la autoestima: autoconcepto, autenticidad y autoeficacia. El grupo que tiene un autoconcepto positivo cuenta con el cultivo de habilidades que inciden en un sentido positivo del self, tanto a nivel personal como profesional. El grupo que tiene un autoconcepto negativo, en cambio, expresa sentimientos de vergüenza, suciedad y baja autoestima, así como un sentimiento de desagrado hacia la prostitución.

El segundo pilar es la autenticidad, entendida como el sentimiento de que uno está actuando con su verdadera personalidad. El grupo que experimenta autenticidad afirma que el trabajo sexual le ha permitido conocerse mejor y que puede conservar su personalidad en el contexto del trabajo. El grupo que expresa inautenticidad, en cambio, siente miedo a que se desvele su identidad a causa de la identidad totalizante del estigma.

El tercer pilar es la auto-eficacia: algunas participantes afirman que la prostitución les ha permitido un estilo de vida autónomo y autodeterminado. Por otro lado, la falta de autoeficacia se da en los sujetos para quienes el trabajo sexual no es una elección y que sienten baja confianza hacia su capacidad de dejarlo.

Radačić (2012) analiza cuáles son los factores responsables del éxito en la organización política de las trabajadoras sexuales de Nueva Zelanda. El primer factor ha sido no hacer diferencias entre sus miembros por posición social o ámbito en el que cada una ejerciera la prostitución; es decir, ver una problemática común que las unía como colectivo. En la misma línea, establecer un sentido de comunidad, con una actitud inclusiva. La pertenencia a este colectivo ha incrementado su autoeficacia, puesto que luchan contra la discriminación y cuentan con un espacio en el que compartir ideas y generar un cambio político. 
Como vemos, los sujetos de esta muestra han llevado a cabo diferentes estrategias: la construcción de una autoimagen colectiva, de un sentido de comunidad y de un proceso de politización, el cual incrementa su capacidad de agencia. Hay que tener también en cuenta que esta transformación social de la que son partícipes va en la dirección de igualarlas al resto de la ciudadanía, puesto que pretende acabar con el estigma de criminalidad. Por lo tanto, las diferencias entre el endogrupo y el resto de la sociedad tienden a disminuir.

El análisis de las distintas muestras demuestra que las estrategias de empoderamiento ayudan a revertir las violencias del estigma y, por tanto, a reducir sus repercusiones psicológicas.

En primer lugar, inciden en el autoconcepto y, por tanto, en la autoestima. Esto lo consiguen al darle un significado positivo al trabajo. Asimismo, cuando emplean un factor de su rol principal -como es la religión- para afrontar una problemática de su rol como trabajadora sexual, están reduciendo el conflicto entre roles. También lo hacen cuando inciden políticamente para que su identidad se desligue de la criminalidad. El cambio de autoconcepto no sólo es individual: las estrategias grupales de apoyo mutuo ayudan a construir un significado colectivo positivo, así como un sentido de pertenencia y, en consecuencia, a romper con el aislamiento social. Este autoconcepto colectivo positivo se puede obtener de dos modos: el primero es, como en el artículo de Weitzer (2017), estigmatizar al exogrupo para normalizar al endogrupo. El segundo es, como en el colectivo de Nueva Zelanda, prescindir de los parámetros del estigma y hallar una definición común positiva, así como una alianza colectiva. Esta unión colectiva tiene lugar en diversas muestras y ayuda a acabar con la violencia.

La construcción de una autoimagen positiva revierte el autoconcepto de persona destruida que comporta el estigma y, por tanto, incentiva las conductas de autocuidado y cuidado mutuo. Es decir: ayuda a cambiar el esquema cognitivo según el cual las trabajadoras sexuales somos meritorias de violencia. La consecuencia, por tanto, es un incremento de la autoeficacia o, en otras palabras, la construcción de un locus de control interno gracias al que los sujetos de las muestras pueden hacer que la violencia disminuya.

\section{Conclusiones}

A lo largo del artículo, se ha analizado cómo el estigma de prostituta puede incidir en la salud mental y generar o cronificar una Depresión, un TEPT o un Trastorno relacionado con las sustancias, mediante sus repercusiones en diversos ámbitos.

El primero es la violencia institucional, la cual se traduce, en primer lugar, en legislaciones punitivistas. Éstas fomentan la clandestinidad e incrementan el riesgo de agresiones, ya sea por el desplazamiento forzoso a lugares aislados o porque la trabajadora no denuncie las agresiones padecidas para no figurar como prostituta. Por lo tanto, la discriminación legal aumenta el riesgo y la violencia. Ésta es la principal causa de los tres trastornos mencionados.

Las agresiones, a las que contribuyen las legislaciones punitivistas, aumentan las experiencias de violencia que vivimos y, por lo tanto, pueden cronificar un TEPT, puesto que contribuirán a que se perpetue la hostilidad del ambiente.

Las instituciones también ejercen violencia mediante el abandono. Esto se traduce de dos maneras: la primera es la falta de derechos laborales, la cual comportará unas malas condiciones de trabajo que incrementan el riesgo de adición a las sustancias. La segunda es la falta de alternativas laborales y de oportunidades, así como la pobreza. Cuando el trabajo sexual es la única opción y, a su vez, supone unas circunstancias adversas -potenciadas por el punitivismo-, el riesgo de padecer o que se cronifique un TEPT aumenta considerablemente. La deshumanización a la que nos aboca el estigma se ve reflejada en la pasividad institucional de cara a nuestras problemáticas. 
El estigma también repercute en la soledad y el aislamiento. La falta de apoyo social puede conllevar relaciones de dependencia, incluso violentas. El modo en que el estigma marca nuestro autoconcepto también puede generar una actitud de pasividad hacia estas violencias, por la idea, inherente al estigma, de que las trabajadoras sexuales las merecemos. Esto puede derivar en ideaciones suicidas, conductas sexuales de riesgo, consumo de sustancias y abandono de los programas de salud.

La falta de apoyo también puede verse reflejada en un trato estigmatizante por parte de organizaciones asistencialistas. Hay que destacar el papel nocivo que puede tener una atención psicológica estigmatizante, revictimizante, psicopatologizante o carente de información.

El estigma construye una autoimagen desempoderante y marcada por la culpa, de persona destruida. Esto disminuye nuestra capacidad de resiliencia, tan necesaria para superar los traumas que puedan dar lugar a un TEPT, o para que las circunstancias adversas que afrontamos, a muchos niveles, no den pie a una Depresión o incidan en el consumo de sustancias.

A lo largo del artículo, pues, se ha analizado la causalidad de las diferentes violencias en la psicopatología, pero también se han analizado las posibilidades de empoderamiento y cómo éste actua como un factor protector. A partir de las muestras estudiadas, se ha señalado que, gracias la definición positiva del autoconcepto, a nivel personal o grupal, las trabajadoras sexuales dejamos de concebirnos como sujetos meritorios de violencia. Esta definición positiva del autoconcepto también incrementa nuestra autoeficacia, por ejemplo, mediante diferentes estrategias de autoprotección y protección grupal. También incrementa nuestro control en nuestras vidas privadas. Es decir: establecemos un locus de control interno.

Este artículo ha perseguido dos finalidades: la primera es analizar qué violencias del estigma de prostituta están relacionadas con la aparición o desarrollo de la psicopatología, a fin de que el profesional de la salud mental los tenga en cuenta durante los tratamientos. En primer lugar, es necesario que, durante el análisis del problema, se tengan en cuenta todas las violencias que puede atravesar una paciente trabajadora sexual. Éstas deberán contemplarse como posibles causas de su comportamiento y sus cogniciones, por un lado, a fin de no establecer conclusiones psicopatologizantes; por el otro, para no dar por sentado que la causa del malestar es el sexo sin deseo, deducción que puede corresponder más al imaginario social sobre prostitución que a las circunstancias reales de la paciente. Asimismo, el tratamiento deberá diseñarse con la finalidad de provocar en la paciente un cambio cognitivo-conductual que le permita incidir en las violencias mencionadas.

Debe tenerse en cuenta, sin embargo, que la violencia institucional queda fuera de su poder. Por ello, en relación a este tipo de violencia, el tratamiento deberá limitarse a los esquemas cognitivos por los que la paciente se culpabiliza de las mismas. En muchos ocasiones, será difícil -cuando no imposible- que la paciente pueda realizar cambios en el ambiente que le permitan evitar estas violencias. El profesional deberá tener esto en cuenta, para no responsabilizar o psicopatologizar a la paciente ante estas dificultades

En consecuencia, el tratamiento deberá centrarse sobre todo en los ámbitos del autoconcepto y las relaciones interpersonales. Deberá trabajar los esquemas cognitivos por los que, a causa del estigma, la paciente se crea meritoria de las violencias ejercidas por su entorno, tanto profesional como personal, y pierda capacidad de agencia y autoeficacia ante las mismas. Se deberá, por tanto, incrementar la autoestima, la autoeficacia y el locus de control interno.

Para finalizar, los profesionales de la salud mental tenemos la responsabilidad ética de señalar qué violencias repercuten negativamente en la salud de la población o, en este caso, de un sector. Urge, 
por tanto, realizar informes acerca del impacto negativo que la violencia institucional ejerce en la salud de las trabajadoras del sexo, a fin de apelar a las instituciones responsables. 


\section{Bibliografía}

American Psychiatric Association (2014) Guía de consulta de los criterios diagnósticos del DSM5.:http://www.eafit.edu.co/ninos/reddelaspreguntas/Documents/dsm-v-guia-consulta-manualdiagnostico-estadistico-trastornos-mentales.pdf Visitado julio 2020

Ayuste, A., Gijón, M., Payá, M., y Rubio, L. (2015). Social work and prostitution: an approach to educational paractices. European Journal of Social Work, 19(2), 204-18.

DOI:10.1080/13691457.2015.1024202

Benoit, C., Jannson, S.M., Smith, M., y Flagg, J. (2018). Prostitution, stigma and its effects on the working conditions, personal lives, and health of sex workers. The journal of research, 55(4-5), 457471. https://doi.org/10.1080/00224499.2017.1393652

Bilardi, J.E., Miller, A.Hocking, J.S., Keogh, L., Cummings, R., Chen, M.Y., Bradshaw, C.S., y Farley, C.K. (2010). The Job Satisfaction of Female Sex Workers Working in Licensed Brothels in Victoria, Australia. The Journal of sexual medicine, 8(116-122).

https://doi.org/10.1111/j.1743-6109.2010.01967.x

Cange, C., Kouanda, S. Ky-Zerbo, O., y Wirtz, A.L. (2019). Effects of traumatic events on sex workers' mental health and suicide intentions in Burkina Fasso: a trauma-informed approach. Sexuality and health. 16(4):348-357. DOI: 10.1071/SH17213

Carlson, C.E., White, S.S., Norcini-Pala, A., Cordisco-Tsai, L., Wainberg, M., y Aira, T. (2017). The Impact of Violence, Perceived Stigma, and Other Work-Related Stressors on Depressive Symptoms Among Women Engaged in Sex Work. Glob Soc Welf 4(2): 51-57. DOI: 10.1007/s40609-017-0085-5

Coetzee, J., Buckley, J., Otwombe, K., Milovanovic, M., Gray, G.E., y Jewkes, R. (2018). Depression and Post Traumatic Stress amongst female sex workers in Soweto, South Africa: A cross sectional, respondent driven sample. PLoSOne, 13(7): e0196759. DOI: 10.1371/journal.pone.0196759

Goffman, E. (1963). Estigma. La identidad deteriorada. Buenos Aires: Amorrortu

Goretti-laisuklang, M., y Ali, A. (2017). Psychiatric Morbidity Among Comercial Sex Workers. Indian Journal of Psychiatry, 59 (4), 465-470. DOI 10. /psychiatry.IndianJPsychiatry_147_16

Ibáñez-Gracia, T., Feliu-i-Samuel-Lajeunesse, J., Maestres-Useche, B., Martínez-Martínez,L.M., PallíMonguilod, C., y Peñaranda-Cólera, M.C.; Pujal-i-Llombart, M.; Tirado-Serrano, F.J. (2016). Fonaments psicosocials del comportament humà. Barcelona: FUOC.

Krumrei-Mancuso, E.J. (2017). Sex Work and Mental Health: A Study of Women in the Netherlands. Arch Sex Behav., 46 (6), 1843-1856. DOI: 10.1007/s10508-016-0785-4 .

Lim, S., Peitzmeier, S., Cange, C., Papworth, E., LeBreton, M., Tamoufe, U., Kamla, A., Billong, S., Fokam, P., Njindam, I., Decker, M.R., Sherman, S.G., y Baral, S. (2015). Violence Against Female Sex Workers in Cameroon. JAIDS Journal of Acquired Immune Deficiency Syndromes, 68, 241-S247. DOI: 10.1097/qai.0000000000000440

Pandiyan, K., Shekar, H.C., Prashanth, N.R., i Gangadara, N. (2014) A Study of Psychological Morbidity among Female Sex Workers . IOSR Journal of Mathematics (IOSR-JM), 10(2), 81-88.

Pinedo-González, R., y López-Sánchez, F. (2015). Soledad social y emocional, factores de riesgo asociados en personas que ejercen la prostitución. Apuntes de Psicología, 33 (1), 17-22.

Puri, N., Shannon, K., Nguyen, P., y Goldenberg, S.M. (2017). Burden and correlates of mental health diagnoses among sex workers in an urban setting. BMC Women's Health, 17 (1) 133. https://doi.org/10.1186/s12905-017-0491-y 
Radačić, I. (2017). New Zealand Prostitutes' Collective-An Example of a Successful Policy Actor. Social Sciences, 6(46). https://doi.org/10.3390/socsci6020046

Rössler, W., Koch, U., Lauber, C., Hass, A.-K., Altwegg, M., Adjacic-Gross, V. y Landolt, K. (2010). The mental health of female sex workers. Acta Psychiatrica Scandinavica, 122(2), 143-152.

Ryan, M., Nambiar, D., y Ferguson, L. (2019). Sex work-related stigma: Experiential, symbolic and structural forms in the health systems of Delhi, India. Social Science and Medicine, 228. DOI: 10.1016/j.socscimed.2019.02.052

Sardana, S., Marcus, M., y Verdeli, H. (2016). Narratives of Violence, Pathology, and Empowerment: Mental Health Needs Assessment of Home-Based Female Sex Workers in Rural India. Journal fo Clinical Psychology, 72(8), 827-838. https://doi.org/10.1002/jclp.22364

Smith, E.M. (2016) 'It gets very intimate for me': Discursive boundaries of pleasure and performance in sex work. Sexualities, 20 (3), 344-363.https://doi.org/10.1177/1363460716665781

Štulhofer, A.; Sinković, M.; Božić, J., i Baćak, V. (2016) Victimization and HIV Risks Among Croatian Female Sex Workers: Exploring the Mediation Role of Depressiveness and the Moderation Role of Social Support. Sage Journals. 23 (1), 67-88. https://doi.org/10.1177/1077801216636241

Truschka, J. (2016). Prostitute-serving Organizations (PSOs) and their Perspectives on Mental Health Concerns Facing Consenting, Adult Prostitutes and other Sex Workers: An International, Qualitative Study Involving New Zealand, Australia, the United Kingdom and the United States / Truschka, Jennifer.Ann Arbor ProQuest Dissertations \& Theses

Vallejo-Pareja, MA(Coord.) (2015). Manual de terapia de conducta. Tomo I (2a. ed.). $\quad$ Madrid: Dykinson

Velez, C., y Audet, C. (2019). Indoor Female Sex Workers' Experiences of Counselling: A Hermeneutical Phenomenological Exploration.Canadian Journal of Counselling and Psychotherapy, 53(4)

Vidal, C.E. (2014). Preditores de prováveis transtornos mentais comuns (TMC) em prostitutas utilizando o Self-Reporting Questionnaire. J. bras. psiquiatr., 63 (3), 205-212. http://dx.doi.org/10.1590/0047-2085000000027.

Yuen W.W.; Wong, W.C.; Holroyd, E.;y Tang, C.S. (2014). Resilience in work-related stress among female sex workers in Hong Kong. Qual Health Res, 24 (9), 1232-41. AIDS Care,28(7), 816-824.DOI: 10.1080/09540121.2016.1139038

Weitzer, R. (2017). Resistance to sex work stigma. Sexualities 21(5-6). https://doi.org/10.1177/1363460716684509

Zendher, M., Mustchler, J., Rössler, W., Rufer, M., y Rüsch, N (2019) Stigma as a Barrier to Mental Health Service Use Among Female Sex Workers in Switzerland. Journal List, Front Psychiatry, 10-32. doi: 10.3389/fpsyt.2019.00032

Zhang, C., Li, X., Su, S., Hong, Y., Tang, Z., y Shen, Z. (2015). Violence against Chinese females sex workers: a hierarchical multiple regression analysis. Health Care Women Int., 36(7), 797-815. doi:10.1080/07399332.2014.909432.

Zhang, L., Li, X., Wang, B., Shen, Z., Zhou, Y., Xu, J., Tang, Z., y Stanton, B. (2017). Violence, Stigma and Mental Health Among Female Sex Workers in China: A Structural Equation Modeling. Women and health, 57(6), 685-704.DOI: 10.1080/03630242.2016.1186781 
Ziao, S., y Bungay, V. (2018). Intersections of Stigma, Mental Health, and Sex Work: How Canadian Men Engaged in Sex Work Navigate and Resist Stigma to Protect Their Mental Health. The Journal of Sex Research, 56(4-5). DOI: 10.1080/00224499.2018.1459446 